\title{
Phytopathology"
}

\section{Time to Fight: Molecular Mechanisms of Age-Related Resistance}

\author{
Lanxi $\mathrm{Hu}$ and $\mathrm{Li}$ Yang ${ }^{\dagger}$
}

Department of Plant Pathology, University of Georgia, Athens, GA 30602

Accepted for publication 10 June 2019.

\begin{abstract}
Plant age is a crucial factor in determining the outcome of a host-pathogen interaction. In successive developmental stages throughout their life cycles, plants face dynamic changes in biotic and abiotic conditions that create distinct ecological niches for host-pathogen interactions. As an adaptive strategy, plants have evolved intrinsic regulatory networks that integrate developmental signals with those from pathogen perception and defense activation. As a result, amplitude and timing of defense responses are optimized, so as to balance the cost and benefit of immunity activation. A general term "age-related resistance" refers to a gain of disease resistance against a certain pathogen when plants reach a relatively mature stage. Age-related resistance is a common observation on fruits, vegetables, and row crops for their resistance against viruses, bacteria, fungi, oomycetes pathogens, and insects. This review focuses on the recent advances in understanding the molecular mechanisms of how plants coordinate developmental timing and immune response.
\end{abstract}

Keywords: genetics and resistance, plant stress and abiotic disorders

An innate immune system protects plants from most pathogens in the environment. Surface localized pattern recognition receptors (PRRs) in plant cells can detect conserved microbial-derived molecules, such as flagellin from bacteria, fungal chitin, and $\beta$-glucans from oomycetes, to activate pattern-triggered immunity (PTI) (Saijo et al. 2018; Zipfel 2014). PTI leads to a spectrum of physiological and biochemical responses to counteract pathogen invasion, including the fortification of cell wall, accumulation of reactive oxygen species (ROS), synthesis of antimicrobial compounds, and transcriptional reprogramming of the phytohormone crosstalk (Saijo et al. 2018). Successful pathogens can subvert PTI by proteinaceous virulence effectors. To execute their virulence function, effectors are delivered into plant cells or the apoplastic space where they interact with and further interfere with or redirect the function of host cellular components, resulting in compromised defense responses, and eventually, pathogen multiplication (Toruño et al. 2016). To counteract the effector-triggered susceptibility, plants have evolved a class of intracellular innate immune receptors known as nucleotide binding-leucine-rich repeat (NLR) receptors, which can recognize the presence of effectors either through direct

${ }^{\dagger}$ Corresponding author: L. Yang; li.yang1@uga.edu

Funding: A portion of this research was supported by a grant from the University of Georgia Research Foundation, Inc.

The author(s) declare no conflict of interest.

(c) 2019 The American Phytopathological Society binding or effector-triggered modifications of host proteins (Cui et al. 2015; Jones and Dangl 2006). The recognition of effectors by NLRs activates effector-triggered immunity (ETI) (Cui et al. 2015; Jones et al. 2016), which often result in a rapid programmed cell death, known as the hypersensitive response (HR), as well as many outcomes that are shared with PTI (Cui et al. 2015; Jones and Dangl 2006).

A positive correlation between host age and disease resistance has been observed in many flowering plants (Develey-Riviere and Galiana 2007; Panter and Jones 2002; Whalen 2005). The term "age-related resistance" (ARR) describes the gain or reinforcement of disease resistance during the process of host maturation. Constitutive or ectopic immune response may lead to deleterious effects such as dwarfism, necrosis, and/or yield loss (Bomblies and Weigel 2007; Brown 2002; Huot et al. 2014), so launching a robust and specific disease resistance response at an optimal time may minimize the potential yield loss caused by pathogen attack and temper negative effects due to unnecessary immunity activation. ARR is a common defense phenomenon observed among economically important fruits, vegetables, and crops generally. Therefore, agriculture practices routinely adapt plant development knowledge in disease management strategy programs. For example, planting date is often adjusted to minimize the cost of exposing plants at a susceptible age to a seasonally active pathogen (Hershman et al. 1990; Hesler et al. 2005; Hunger et al. 1992; Moschini and Pérez 1999). ARR traits are targets of breeding programs to enhance disease resistance (Huang and Röder 2004; Singh et al. 2005; Steffenson et al. 1996). Distinct modes of agedependent host-microbe interactions are covered under this general 
term. Based on the heterogeneity of host species, host age, infected organ, and causal pathogens, ARR may also be referred to as "ontogenic resistance," "developmental resistance," "mature seedling resistance," or "adult plant resistance" (Develey-Riviere and Galiana 2007; Panter and Jones 2002; Whalen 2005). The complexity of nomenclature indicates that there are multiple mechanisms involved in developmental acquired defense.

An essential parameter of ARR is plant age. However, precisely quantification of plant age is not always straightforward. Plant age may refer to the developmental progression of individual organs (e.g., their size, color, and/or shape), known as ontogenesis (Fig. 1A). Plant organs undergo cell division, cell expansion, differentiation, ripening in the case of fruit, and finally, senescence. Leaves and fruits at early developmental stages are often protected by surrounding structures such as preexisting leaves, sepals or petals, which reduces the risk of pathogen attack. Elevated disease resistance in mature organs could be an adaptive strategy that minimizes the risk of investment loss (Glander et al. 2018). For example, strawberry fruits and leaves show ontogenic resistance against powdery mildew Podosphaera aphanis (Asalf et al. 2014). When inoculated at bloom or green stages, the fruits are susceptible, but full resistance is apparent only in white and pink stages of strawberries. When strawberry leaves are inoculated at a late development stage, the pathogen also produce less conidia than on early stage leaves (Asalf et al. 2014). Ontogenesis always occurs in the context of the whole plant maturation, so inevitably, ontogenic resistance may be influenced by the chronological and physiological age of the whole plant.

Chronological age of a plant can be defined by the exact amount of time post planting or post organogenesis, such as "weeks after planting" or "days after pollination" (Develey-Riviere and Galiana 2007). The timing of ARR activation as well as progression of plant maturation are heavily influenced by environmental conditions. For example, under short day conditions ( $9 \mathrm{~h}$ of light and $15 \mathrm{~h}$ of darkness), Arabidopsis thaliana (hereafter Arabidopsis) gains enhanced resistance against the bacterial pathogen Pseudomonas syringae 6 weeks post germination, while under long day conditions (16 h of light and $8 \mathrm{~h}$ of darkness), ARR was activated three weeks after germination (Rusterucci et al. 2005). Hence, in addition to chronological age, assessing physiological age of a plant is also useful in determining the timing of ARR onset.
Physiological age of a plant can be defined by the appearance of characteristic morphological and physiological features, such as "flowering stage" or "adult vegetative stage" (Huijser and Schmid 2011; Poethig 2013) (Fig. 1B). Most flowering plants go through successive developmental transitions in a predictable temporal pattern. The first transition is from the embryonic stage to the juvenile vegetative stage, which is characterized by seed germination. Next, plants grow from the juvenile vegetative stage to the adult vegetative stage, which is associated with heteroblasty, gain of flowering competence, reduction of rooting ability, and alteration of epidermal traits (Poethig 2013). A shoot further enters into reproductive stage that eventually results in production of an inflorescence and floral organs. In many species, the meristem undergoes another change of identity from an inflorescence meristem that generates axillary buds to a floral meristem that generates floral organs (Wagner 2017). ARR is often associated with transitions between these developmental stages. ARRassociated with a transition from the embryonic stage to the juvenile vegetative stage is exemplified by distinct interactions between an oomycete and two Brassicaceae species. In the first interaction, cotyledons from Arabidopsis ecotype Col-0 are fully susceptible to downy mildew Hyaloperonospora arabidopsidis isolate Emco5, but true leaves exhibit resistance (McDowell et al. 2005). This postembryonic resistance is controlled by a single locus RPP31, although the causal gene is still unknown (McDowell et al. 2005). In the second interaction, $H$. parasitica is virulent on cotyledons but not the true leaves of two broccoli cultivars (Coelho et al. 2009). Many studies have documented ARR against multiple pathogens after a transition from the juvenile to the adult vegetative phase. For instance, adult leaves of Arabidopsis are more resistant to fungal (Sclerotinia sclerotiorum) and bacterial (Xanthomonas oryzae and Pseudomonas syringae) pathogens than are juvenile leaves (Xu et al. 2018). In maize, the adult stage is also associated with high resistance to common rust (Puccinia sorghi), European corn borer (Ostrinia nubilalis), and Southern leaf blight (Cochliobolus carbonum) (Abedon and Tracy 1996; Marla et al. 2018). Similarly, floral transition triggers age-dependent resistance; Nicotiana tabacum gains resistance to Phytophthora parasitica and H. tabacina after switching to the flowering stage (Wyatt and Kuc 1992).

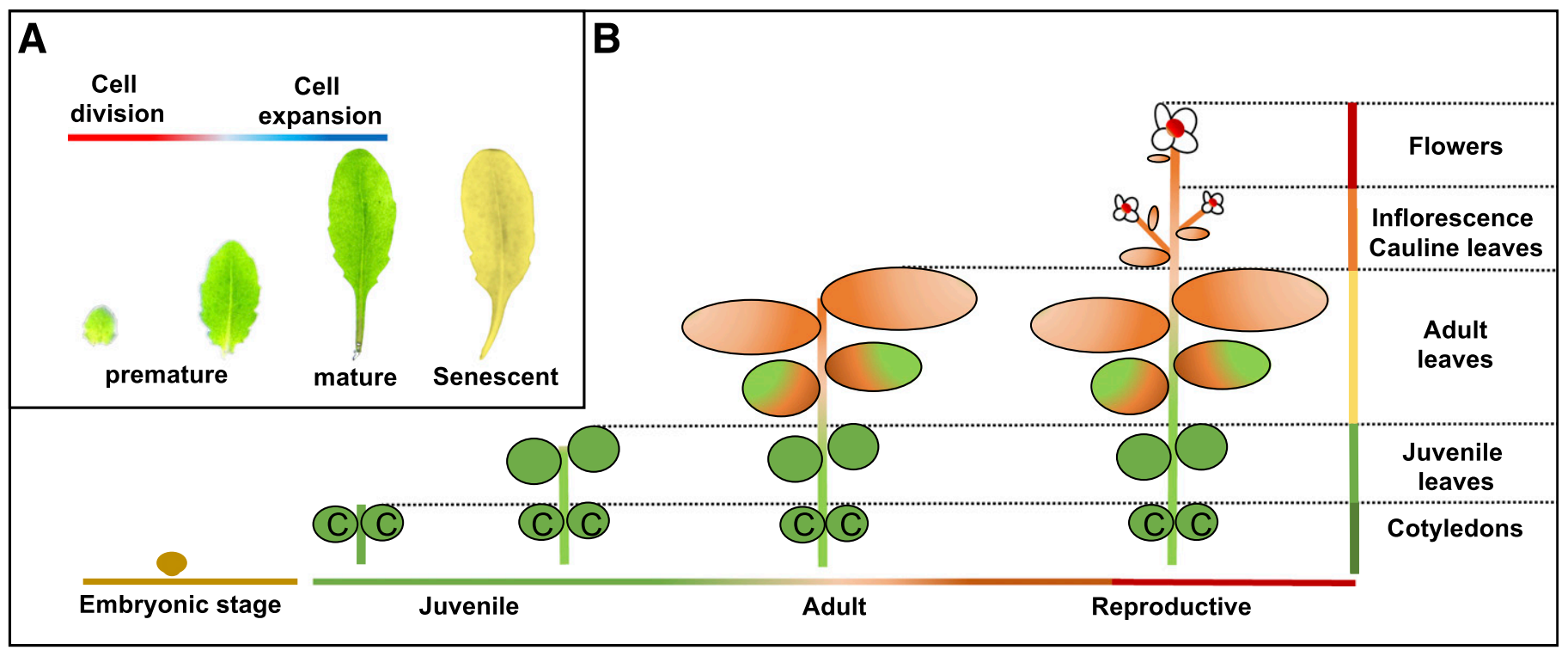

FIGURE 1

Diagram of organ maturation and successive developmental stages of a plant. A, Leaf maturation in Arabidopsis. B, The progression of shoot maturation. Capitalized " $\mathrm{C}$ " refers to cotyledon. Mature organs color-coded in orange. 
Despite the potential value to agriculture, our knowledge of the molecular mechanisms of ARR is limited. Key current questions include the following. (i) What are the reliable molecular markers of plant age for predicting the timing of ARR? (ii) How do plants integrate developmental timing and defense activation at a molecular level? (iii) Do pathogens evolve virulence factors to counteract ARR? (iv) If so, how do they suppress ARR? (v) Is it possible to decouple the pace of developmental progression and the onset of ARR? (vi) What are potential tradeoffs caused by precocious activation of ARR? Studying the molecular mechanisms of ARR could be challenging due to complex interactions between environmental conditions, plant physiology and pathogen life cycle. The plasticity of morphological traits under varying environmental conditions (temperature, humidity, and UV exposure, etc.) complicates the quantification of "plant age." Distinct environmental conditions associated with each developmental stage may influence the outcome of disease resistance in addition to the action of an age-dependent immune response. For many plants, genetic tools that distinguish age-dependent innate immune response from secondary consequence caused by physiological and morphological changes, such as trichome density, cuticle thickness, and leaf curling, associated with developmental transitions are still limited. There is a growing body of evidence demonstrating that ARR is controlled by a sophisticated intrinsic regulatory network that directs plants to adjust their immunity for predictable changes of biotic stress occurring during their life cycles.

A summary of ARR observations is provided in Table 1. Only examples with known candidate causal genes are listed. For a more comprehensive summary of ARR events, please refer to these excellent reviews (Develey-Riviere and Galiana 2007; Panter and Jones 2002; Whalen 2005).

\section{MOLECULAR CONNECTIONS BETWEEN DEFENSE RESPONSE AND DEVELOPMENTAL TIMING}

ARR associated with organ maturation. Recently, genes and pathways associated with ontogenic resistance are identified by transcriptome analysis in Arabidopsis, apple, strawberry, and cucumber (Ando et al. 2015; Gusberti et al. 2013; Mansfeld et al.
2017; Zou et al. 2018). These studies shed light on putative signaling cascades integrating developmental timing and the onset of ontogenic resistance. In Arabidopsis, resistance against Pseudomonas syringae is established between two and six days after germination (Zou et al. 2018). In 2-day-old seedlings, high levels of TARGET OF EARLY ACTIVATION TAGGED 1 and 2 (TOE1 and TOE2) proteins suppressed the transcription of FLAGELLINSENSITIVE 2 (FLS2) by binding to the A/T rich motif in its promoter, TOE binding site in the FLS2 promoter (TBSF). FLS2 encodes a leucine-rich repeat serine/threonine protein kinase that acts as a PRR to activate plant immunity after recognizing the bacterial PAMP flg22, a 22 amino acid epitope in a flagellin protein (Zipfel et al. 2004). In 6-day-old seedlings, increased expression of microRNA172 (miR172) repressed TOE1/2 transcripts, thereby relieving the suppression of $F L S 2$ by TOE1/2. Hypersensitivity to flg22 was observed in the toe 1 toe 2 double mutant and in plants overexpressing miR172, confirming their roles in regulating FLS2mediated PTI. Another PRR, EF-TU RECEPTOR (EFR), which recognizes the bacterial elongation factor EF-Tu, was also suppressed by TOE1/2, suggesting that these two transcription factors regulate PTI triggered by multiple PAMPs. Importantly, MIR172 precursors were activated by flg22 treatment. Therefore, the miR172-TOE1/2 module acts as an integrator of environmental and developmental cues to control the onset of PTI during cotyledon ontogenesis (Fig. 2A).

The miR172-TOE1/2 module is well documented as a regulator of vegetative phase change and flowering (Zhu and Helliwell 2010). MiR172 was induced by flg22 in both 2-day-old and 8-week-old plants (Zou et al. 2018). However, no changes in miR172 accumulation was found after flg22 treatment (Li et al. 2010) or Pseudomonas syringae infection (Zhang et al. 2011) using 4- to 5week-old plants, suggesting that the upregulation of miR172 by PAMP is probably age-dependent in previous studies. Pathogeninduced alteration of miR172 accumulation has been observed in mulberry infected with yellow dwarf disease, grapevine infected with grapevine leafroll disease and rice infected with the blast fungus, Magnaporthe oryzae (Alabi et al. 2012; Gai et al. 2014; Li et al. 2014). Plants used in these studies were beyond the cotyledon stage, so the temporal regulation of miR172 is likely to control ARR

TABLE 1

A summary of age-dependent defense responses with candidate genes

\begin{tabular}{|c|c|c|c|c|}
\hline Host & Pathogen & $\begin{array}{l}\text { Developmental } \\
\text { transitions }\end{array}$ & Genes involved & Reference \\
\hline Maize & $\begin{array}{l}\text { Common rust and European corn } \\
\text { borer } \\
\text { Cochliobolus carbonum }\end{array}$ & $\begin{array}{l}\text { Juvenile to adult } \\
\text { Juvenile to adult }\end{array}$ & $\begin{array}{l}\text { Corngrass1 } \\
\text { (miR156) } \\
\text { Hm1 weak allele }\end{array}$ & $\begin{array}{l}\text { (Abedon and Tracy 1996) } \\
\text { (Marla et al. 2018) }\end{array}$ \\
\hline Rice & Xanthomonas oryzae & Juvenile to adult & $X a 21$ & (Century et al. 1999) \\
\hline Arabidopsis & $\begin{array}{l}\text { Pseudomonas syringae } \\
\text { Hyaloperonospora parasitica } \\
\text { Pseudomonas syringae } \\
\text { Insects } \\
\text { Pseudomonas syringae }\end{array}$ & $\begin{array}{l}\text { Adult plant maturation } \\
\text { Postembryonic to } \\
\text { juvenile } \\
\text { Ontogenic resistance } \\
\\
\text { Juvenile to adult } \\
\text { Vegetative to flowering }\end{array}$ & $\begin{array}{l}\text { SVP, SOC1 } \\
\text { RPP13 } \\
\text { MiR172, TOE1, } \\
\text { TOE2 } \\
\text { SPL9 } \\
\text { HAE, HSL2; IDA, } \\
\quad \text { NEV } \\
\end{array}$ & $\begin{array}{l}\text { (Wilson et al. 2017) } \\
\text { (McDowell et al. 2005) } \\
\text { (Zou et al. 2018) } \\
\text { (Mao et al. 2017) } \\
\text { (Patharkar et al. 2017) }\end{array}$ \\
\hline Tomato & $\begin{array}{l}\text { Phytophthora infestans } \\
\text { Cladosporium fulvum }\end{array}$ & $\begin{array}{l}\text { Vegetative to flowering } \\
\text { Vegetative to flowering }\end{array}$ & $\begin{array}{l}p h-3 \\
\text { Cf-9B }\end{array}$ & $\begin{array}{l}\text { (Zhang et al. 2014) } \\
\text { (Panter et al. 2002) }\end{array}$ \\
\hline $\begin{array}{l}\text { Nicotiana } \\
\text { benthamiana }\end{array}$ & Pseudomonas syringae & Vegetative to flowering & NbCSPR & (Saur et al. 2016) \\
\hline Barley & Powdery mildew & Adult plant resistance & HVRBOHF2 & (Torres et al. 2017) \\
\hline
\end{tabular}


associated with different developmental processes. This is further corroborated by a study in Solanum lycopersicum, where overexpression of miR172 precursors enhanced resistance to Phytophthora infestans at the five to six leaf stage (Luan et al. 2018).

Strengthening physical barriers and chemical defense contribute to ontogenic resistance as well as activation of innate immunity in cucumbers and strawberries (Ando et al. 2015; Gusberti et al. 2013; Mansfeld et al. 2017). Cucumber fruits show ontogenic resistance against the oomycete pathogen Phytophthora capsici (Ando et al. 2015). Young fruits at a rapidly elongating stage were most susceptible, resistance was developed when cucumbers reached maturity. Comparing the transcriptome of peel or exocarp isolated from susceptible (8 days postpollination) and resistant (16 days postpollination) fruits revealed candidate genes associated with ARR against Phytophthora capsici in cucumber (Ando et al. 2015). These genes represent pathways regulating cuticle synthesis, flavonoid biosynthesis, oxidative stress as well as PTI and ETI, suggesting that ARR is composed of coordinated reinforcement in physical barriers, chemical defense and innate immunity (Ando et al. 2015). In another study, researchers compared the transcriptomic and metabolomic profile of peels from an ARR-capable (Vlaspik) and an ARR-defective (Gy14) cucumber cultivar at susceptible and resistant stages. The most abundant compounds uniquely presented in peel extracts from the ARR-capable cultivar at the resistant age were the terpenoid glycosides (Mansfeld et al. 2017). The role of these compounds in ARR against Phytophthora capsici is an intriguing question for future research. When comparing the transcriptomes between the young and old apple leaves inoculated with Venturia inaequalis, a gene encoding the homolog to Arabidopsis ENHANCED DISEASE SUSEPTIBILITY 1 (EDS1), a positive regulator of basal defense and ETI, was found to be down-regulated in old leaves (Gusberti et al. 2013). In contrast, an EDS1-like gene was preferentially expressed during ontogenic resistance in mature cucumber (Ando et al. 2015). These observations suggest that modulating the EDS1-regulated immune response may serve as a common strategy in ontogenic resistance. Given the distinct developmental and physiological status between exocarps, peels and leaves used in the cucumber and apple studies, it is intriguing to dissect the shared and tissue-specific components of ontogenic resistance.

ARR associated with vegetative phase change. The microRNA156/157 (miR156/157) is a conserved master regulator of vegetative phase change (Fouracre and Poethig 2016; He et al. 2018; Poethig 2013). From herbaceous to woody plants, the onset of the vegetative phase change is tightly associated with a reduction of miR156/157 level (Poethig 2013). The mature miR156 and miR157 differ in three nucleotides and have comparable functions in suppressing the expression of SQUAMOSA PROMOTER BINDING PROTEIN-LIKE (SPL) transcription factors (He et al. 2018; Rhoades et al. 2002). SPL proteins share a highly conserved 76 amino acids SBP box that is involved in both nuclear import and DNA binding (Birkenbihl et al. 2005; Liang et al. 2008). In the juvenile phase, MIR156/157 genes are expressed at a high level, which suppresses the accumulation of $S P L$ transcripts and proteins (He et al. 2018; Poethig 2013). The expression of MIR156/157 is gradually turned off during shoot maturation, allowing accumulated $S P L$ proteins to specify adult traits. In both dicots and monocots, overexpression of MIR156/157 precursors extends the time window to produce juvenile traits, while plants with reduced level or function of miR156/157 and plants expressing the resistant version of SPLs (rSPLs) to miR156/157 show early phase change (Poethig 2013). In most genomes, miR156/157 targets multiple members in the SPL gene family. They have overlapping and distinct functions in controlling a spectrum of morphological and physiological traits (Wang and Wang 2015). The Arabidopsis genome contains 17 SPL genes, 11 of them $(S P L 2,3,4,5,6,9,10,11,13 A / B, 15)$ are repressed by miR156/157 via mRNA cleavage and/or translational repression (Cardon et al. 1999; Rhoades et al. 2002).

The first genetic evidence linking miR156 levels to ARR came from a study related to resistance of maize to common rust and European corn borer (Abedon and Tracy 1996). MiR156 overaccumulates in the Corngrass $1(\mathrm{Cg} /)$ mutant due to a transposon insertion in the promoter region of zma-miR156b/c locus (Chuck et al. 2007). The $c g 1$ mutant extends the period of the susceptibility window as well as other juvenile traits in maize (Abedon and Tracy 1996; Chandler and Tracy 2007). In contrast, knocking down miR156 activity by target mimicry in rice reduced the number of tillers, rate of leaf initiation (Xie et al. 2012) and also enhanced resistance to rice brown planthopper (BPH, Nilaparvata lugens Stål) (Ge et al. 2018). However, BPH did not show a clear pattern of preference of plant age within tested cultivars (Baqui and Kershaw 1993), so it is still an open question whether endogenous miR156 contributes to ARR against BPH.

Characterization of individual miR156/157-targeted SPL genes revealed how temporal reduction of miR156/157 during vegetative phase changed crosstalks with plant immune responses (Fig. 3). In tobacco, NbSPL6 promoted resistance to Tobacco mosaic virus (TMV) by physically interacting with the Toll-interleukin-1 (TIR) type NLR immune receptor, N (Padmanabhan et al. 2013). Such interaction only occurred in the presence of the defense-eliciting TMV-p50-U1 effector. The gene homolog of NbSPL6 in Arabidopsis, AtSPL6, positively regulated race-specific resistance against Pseudomonas syringae effector avrRps4. Arabidopsis plants with reduced AtSPL6 due to overexpressing miR156 or RNAi were more susceptible to Pseudomonas syringae carrying avrRps4, but not to either avrRpt 2 or avrRpm1 effectors (Padmanabhan et al. 2013). Therefore, SPL6 positively regulates a branch of ETI. It is unknown yet whether NtSPL6 contributes to the observed age-related pattern against TMV in tobacco (Ross 1961). Although AtSPL6 expression is repressed by miR156 (Xu et al.2016), there is no evidence to suggest that ETI against avrRps4 is age-dependent. Future work is needed to validate that temporal expression of SPL6 indeed contributes to age-dependent ETI.

\footnotetext{
FIGURE 2

Age-dependent regulation of FLAGELLIN-SENSITIVE 2 (FLS2)-mediated pattern-triggered immunity (PTI) during plant maturation. A, Onset of FLS2-mediated PTI during cotyledon maturation. Triangle bars indicate gene expression level. TOE1/2 binding motif upstream of FLS2 promoter region is highlighted in magenta. B, Suppression of FLS2 expression by LFY in floral transition. LFY binding motif upstream of FLS2 promoter is highlighted in orange.
}

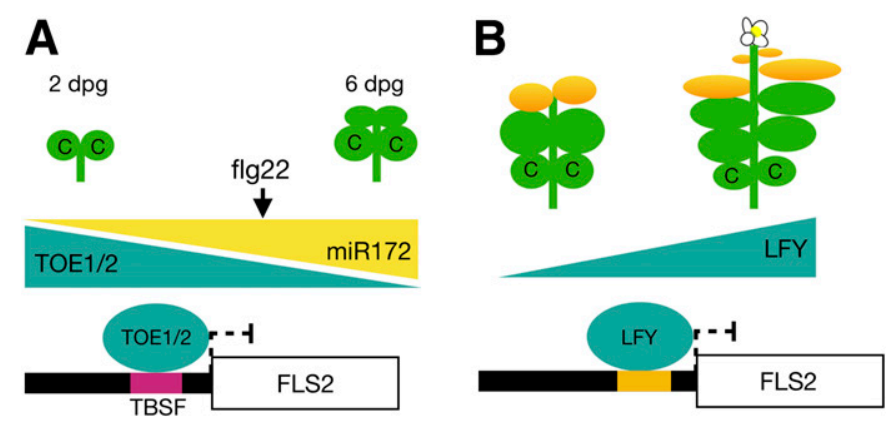


Members of the SPL transcription factor family interconnect phytohormone-mediated defense response and temporal expression of miR156. High levels of SPL9 in the adult stage of Arabidopsis attenuated jasmonic acid (JA) response by stabilizing JASMONATE-ZIM-DOMAIN PROTEIN 3 (JAZ3), a suppressor of JA signaling pathway (Mao et al. 2017). Consistently, bioactive jasmonoyl-isoleucine levels and the expression of JA biosynthesis genes were significantly decreased in rice with reduced miR156 abundance (Ge et al. 2018). However, Arabidopsis at the adult stage is more resistance to both the lepidopteran generalist Helicoverpa armigera and the specialist Plutella xylostella, which is contradictory to the observed low JA response in adult tissue (Howe and Jander 2008; Mao et al. 2017). Instead, it was found that importing of glucosinolates from preexisting leaves contributes to the increased insect herbivore resistance in adult plants (Mao et al. 2017). Such elevated resistance was still observed in plants overexpressing miR156 or expressing rSPL9 (Mao et al. 2017). The authors proposed that constitutive accumulation of defense compounds compensates for the age-related attenuation of JA response (Mao et al. 2017). This proposition partially explains the enhanced resistance to insect herbivory, but the question remains: what is the biological significance of attenuated JA response in the adult plant stage? JA antagonizes salicylic acids (SA)-mediated defense against biotrophic or hemibiotrophic pathogens (Robert-Seilaniantz et al. 2011). It is conceivable that the SPL9-mediated suppression of the JA response may contribute to ARR against biotrophic or hemibiotrophic pathogens by allowing high amplitude of SA response in the adult stage. Plants expressing rSPL9 accumulate high levels of ROS and the transcripts of basal SA responsive genes (Yin et al. 2019), but whether these phenotypes are due to a suppression of JA response by SPL9 remains to be tested. In rice, overexpressing Ideal Plant Architecture 1 (IPA1)/OsSPL14 and OsSPL7 enhanced resistance to bacterial blight (Xanthomonas oryzae pv. oryzae) by reducing gibberellin (GA)-mediated disease susceptibility (Liu et al. 2019). IPA1 and OsSPL7 both physically interact with and further stabilize SLR1, a DELLA transcriptional repressor of GA signaling (Liu et al. 2019). Cumulatively, evidence indicates that diversified immune function of individual SPLs translate temporal decrease of miR156 into the coordinated onset of age-related defense during vegetative phase change.
Potential events downstream of miR156/157-controlled ARR are uncovered by comparing the transcriptome of juvenile and adult tissues. Beydler et al. compared the transcriptomes of juvenile and adult leaf primordia from maize and found that SA and JA pathways were up-regulated in juvenile primordia together with genes involved in oxidative stress response and retrograde redox signaling (Beydler et al. 2016). This study provided candidate genes potentially important for ARR associated with vegetative phase change in maize. Nevertheless, it should be noted that the comparison was done in leaf primordia in the absence of pathogen attack. It is yet unclear how these differences in gene expression may contribute to documented ARR against common rust and the European corn borer in mature leaves (Abedon and Tracy 1996). In Arabidopsis, genes involved in glucosinolate biosynthesis and metabolism, oxalic acid catabolism, ROS accumulation and systemic acquired resistance (SAR) were differentially expressed in fully expanded juvenile and adult leaves from 8-week-old plants (Xu et al. 2018). Although no pathogen inoculation was used, this study offers a picture of differentiated gene expression in the leaf stage where actual infection occurs. It is noteworthy that a systematic search of miR156 binding sites in grape (Vitis vinifera) berries identified an NLR gene as a candidate target of miR156f/g/I (Cui et al. 2018). Thus, the miR156-regulated defense pathway may extend beyond the action of SPLs. It is reasonable to speculate that multiple defense pathways differentially activated in the juvenile and adult stages of plants collectively contribute to the observed broad-spectrum resistance associated with vegetative phase change.

ARR associated with floral transition. It has been a longstanding question whether ARR associated with floral induction is merely a physiological consequence of flowering or an independent program. Recent studies using mutants in flowering genes clearly demonstrate that the floral transition is not linked to the onset of ARR (Lyons et al. 2015; Wilson et al. 2013). Photoperiod-induced transient expression of FLOWERING LOCUS T (FT) triggered early flowering in short-day grown Arabidopsis, but the timing of ARR competence against Pseudomonas syringae was not affected (Wilson et al. 2013). On the other hand, in late flowering mutant, constans-9 (co-9), ARR response was activated at the same time as in wild type (Wilson et al. 2013). In another case, deleting FLOWERING LOCUS C (FLC) in late flowering mutants accelerated flowering without altering resistance against Fusarium

\section{$\operatorname{miR} 156$}
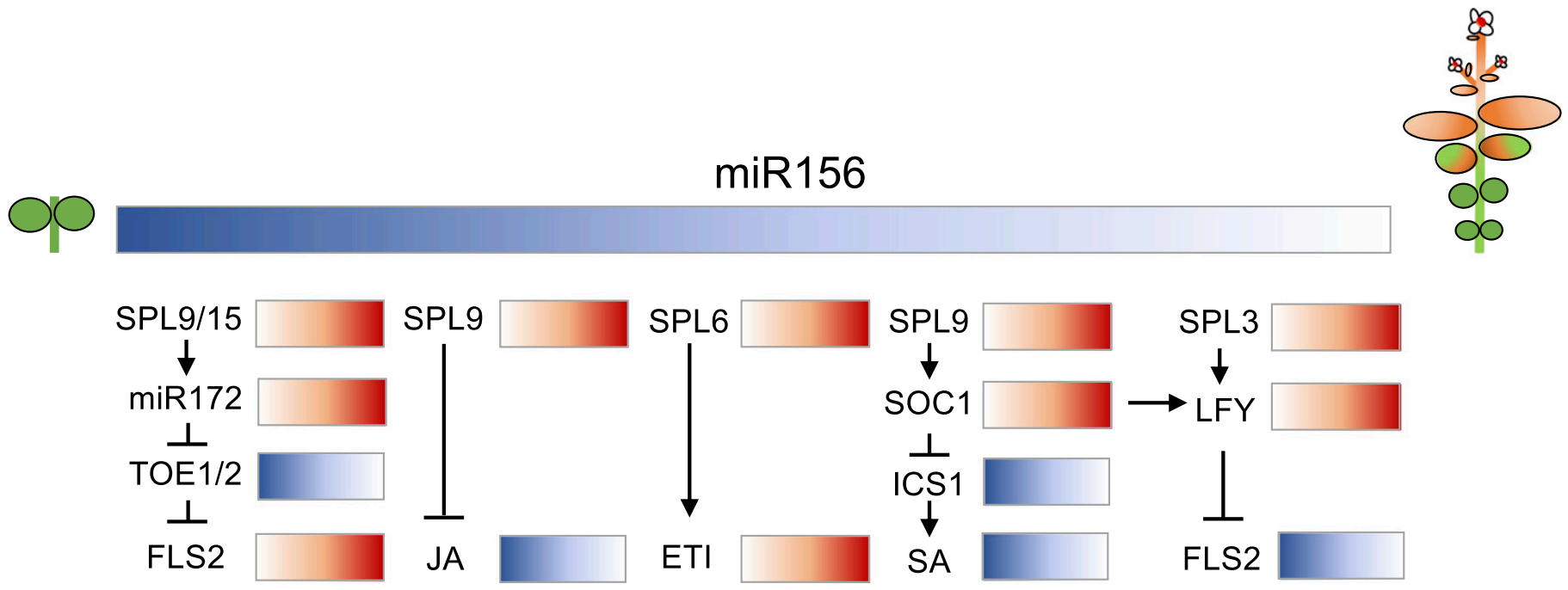

FIGURE 3

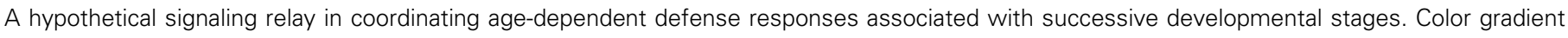

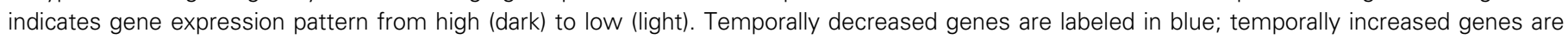
labeled in red. 
oxysporum (Lyons et al. 2015). Although a positive covariation between immunity-related gene expression and flowering time was observed in a natural population of 138 Arabidopsis accessions from Sweden (Glander et al. 2018), this positive covariation can be genetically separated using a segregating recombinant inbred population (Glander et al. 2018). Taken together, the available genetic evidence supports that the molecular programs regulating the timing of ARR onset and the floral transition are two independent pathways.

The study of SHORT VEGETATIVE PHASE (SVP)'s function in ARR and floral transition provides an elegant example of how this protein coordinates these two functions (Wilson et al. 2017). SVP represses flowering by integrating vernalization and themoresponsive pathways (Liu et al. 2009). In addition to early flowering, svp mutants are defective in ARR activation due to attenuated intercellular SA accumulation in mature plants (Wilson et al. 2013; Wilson et al. 2017). Expressing the SVP gene under a meristem specific promoter rescued the flowering time phenotype in svp loss-of-function mutant, but not the ARR defects, demonstrating that the leaf pool of SVP protein is responsible for activating defense and ARR is not a secondary physiological consequence of floral induction (Wilson et al. 2017). In leaves, SVP promoted SA accumulation by repressing SUPPRESSOR OF OVEREXPRESSION OF CO 1 (SOC1), a positive regulator of floral induction. SOC1 repressed the promoter activity of ISOCHORISMATE SYNTHASE 1 (ICS1), a key enzyme in SA biosynthesis, and SA accumulation was reduced in a SOC1 gain-offunction mutant (Wilson et al. 2017; Zheng et al. 2015). In addition, SOC1 may also negatively regulate immunity by promoting stomatal opening (Kimura et al. 2015). Stomatal closure is a key defense mechanism in blocking pathogen entrance (Melotto et al. 2006). Constitutively open-stomata were observed in transgenic plants overexpressing SOC1-GFP in guard cells, and light-induced stomatal opening was significantly suppressed in the socl mutant (Kimura et al. 2015). Thus, tissue specific function of SVP and SOC1 may explain how flowering genes regulate the timing of ARR.

$L E A F Y(L F Y)$ is another dual regulator of flowering time and defense response (Winter et al. 2011) (Fig. 2B). LFY integrates signals that promote flowering and the transition from inflorescence meristem to floral meristem (Moyroud et al. 2010). A genome-wide search revealed that LFY bound to the promoter of FLS2, and repressed its activity (Winter et al. 2011). Flg22-triggered callose deposition was suppressed by inducing $L F Y$ expression in 9-dayold Arabidopsis seedlings (Winter et al. 2011), indicating that LFY directly suppresses PTI output. In addition, cauline leaves from the lfy mutant were more resistance to Pseudomonas syringae (Winter et al. 2011). The authors proposed that LFY redirects plant resources from defense responses to flower and fruit development in order to maximize reproductive fitness (Winter et al. 2011).

Despite the fact that FLS2-mediated PTI is suppressed in cauline leaves due to a high level of LFY (Winter et al. 2011), cauline leaves are still more resistant to Pseudomonas syringae than rosette leaves (Xu et al. 2018). A compensatory mechanism must exist to enhance resistance in cauline leaves. Although such a mechanism has not been discovered, a recent report showed that cauline leaves of Arabidopsis use shedding as a stage-specific defense mechanism (Patharkar et al. 2017). Arabidopsis cauline leaves shed 2 days after being infected with Pseudomonas syringae, which was suggested to be a defense response to eliminate infected tissue and prevent pathogen spread. Unlike cauline leaves, rosette leaves infected with Pseudomonas syringae did not shed (Patharkar et al. 2017). Therefore, shedding is an age-dependent defense response associated with flowering. The shedding of cauline leaves requires genes involved in floral organ abscission and genes involved in SA accumulation and signaling (Patharkar et al. 2017), indicating an integration of a defense signaling pathway (SA response) into a local physiological response (floral organ abscission).
ARR in successive developmental transitions. A continuous increase of resistance against a pathogen can occur in successive developmental stages throughout shoot maturation (Xu et al. 2018). The adult rosette leaves on Arabidopsis are more resistant to Pseudomonas syringae than juvenile leaves, while cauline leaves gain further resistance compared with adult leaves (Xu et al. 2018). Priming of defense by accumulative experience of biotic stresses could be an explanation for the reinforcement of defense in successive stages. Alternatively, components of ARR may coordinate a signaling relay to strengthen/weaken defense response in successive stages (Fig. 3). As detailed above, the miR172-TOE1/2 module promotes ontogenic resistance in postembryonic seedlings by regulating FLS2 expression (Zou et al. 2018). In the transition from the juvenile to the adult vegetative phase, SPL9 (Wu et al. 2009) and SPL15 (Hyun et al. 2016) directly bind to miR172b promoter and activate its expression. It is reasonable to speculate that the FLS2 promoter is derepressed during vegetative phase change and flowering due to the reduction of TOE1/2. On the other hand, SPL3 is a direct activator of LFY in the meristem (Yamaguchi et al. 2009), which may lead to a suppression of FLS2. Therefore, a signaling relay from miR156 to FLS2 may regulate the age-related change of FLS2-mediated PTI associated with seedling ontogeny, vegetative phase change and floral transition. SOC1 is another candidate to relay ARR from the vegetative phase change to the flowering stage. SOC1 suppressed the accumulation of intercellular SA by negatively regulating ICS1, which is important for the onset of ARR in Arabidopsis in a time window between 5 to 8 weeks (Wilson et al. 2017). SOC1 transcription is directly activated by SPL9, so it can potentially relay signals from temporal expression of miR156 to SA accumulation (Wang et al. 2009). SOC1 also forms a protein complex with AGAMOUS-LIKE 24 (AGL24) to activate $L F Y$ expression (Liu et al. 2008). Therefore, SOC1 may serve as a hub to suppress different aspects of age-dependent defense response by integrating developmental signals.

It should be noted that the speculated signaling relays mentioned above are based on epistasis studies from different developmental processes. For example, AGL24 is predominantly expressed in shoot apical meristem (Liu et al. 2008), so it is unclear whether the SOC1-AGL24 interaction can influence defense response occurring in leaves. Similarly, the activation of $L F Y$ by SPL3 was only documented in the shoot apical meristem (Yamaguchi et al. 2009), and further study is needed to validate the function of SPL3 in defense.

\section{SUPPRESSION OF ARR BY PATHOGENS}

Pathogens have evolved a repertoire of virulence tools including toxins, hormone mimics and effector proteins to attenuate the host immune system or redirect nutrition distribution, which eventually promotes their multiplication. Conceivably, ARR could be a target of these pathogen-derived virulence factors. Pathogen infection can dramatically alter developmental timing of a host. Infection with the bacterial pathogens Pseudomonas syringae and Xanthomonas campestris, the oomycete pathogen Peronospora parasitica, and the root-infecting fungal pathogen Fusarium oxysporum accelerated flowering in Arabidopsis (Korves 2003). It is debatable whether this early flowering is a disease symptom caused by stress or a consequence associated with activation of ARR. Recent advances in computational prediction and functional studies on virulence effectors from bacteria, fungi, and oomycetes reveal how pathogens might manipulate plant ARR pathways.

The effector SAP11 from the phytoplasma strain aster yellow witches'-broom delayed flowering when heterologously expressed in Arabidopsis, which was correlated with an altered expression of genes controlling flowering and ARR (Chang et al. 2018). For example, miR156 over-accumulated in SAP11 transgenic plants (Chang et al. 2018). It is hypothesized that delayed flowering is 
beneficial for phytoplasmas and their insect vectors because it prolongs the susceptible time window for colonization. Alternatively, SAP11 may weaken immunity by manipulating key components required for ARR competence and flowering. Another effector PHYL1 from peanut witches'-broom phytoplasma may directly or indirectly interfere with the miR396-mediated decay of SVP transcripts (Yang et al. 2015). Transgenic Arabidopsis expressing PHYL1 showed up-regulation of SVP as well as low miR396 level compared with wild type (Yang et al. 2015).

Large scale yeast-two-hybrid experiments have been carried out to identify host proteins that can interact with effectors or candidate effectors from Pseudomonas syringae, H. arabidopsidis, and Golovinomyces orontii (Mukhtar et al. 2011; Weßling et al. 2014). In these screens, SOC1 was identified as a target of HaRxL45, a candidate effector from $H$. arabidopsidis. Transgenic Arabidopsis expressing HaRxL45 enhanced the susceptibility to Pseudomonas syringae (Fabro et al. 2011). One could speculate that HaRxL45 may have evolved as a virulence factor to suppress the onset or amplitude of ARR by stabilizing SOC1 or promoting its function. TOE2 was also found to interact with multiple effectors from Pseudomonas syringae, namely avrPto, HopBB1, and HopR1 (Mukhtar et al. 2011). These discoveries open a door to study pathogen manipulation of host ARR.

Rhodococcus fascians infection exemplifies how a pathogen interferes with host hormone response to weaken ontogenic resistance. Rhodococcus fascians infection causes foliar distortion, witches'-broom, and leaf galls in various plants (Vereecke et al. 2000). The infected leaves and cotyledons are maintained at a young developmental stage (Depuydt et al. 2009), likely from the effect of cytokinin synthesized by the pathogen (Pertry et al. 2009). Infected cotyledons were shown to be maintained as a sink tissue for pathogen colonization (Dhandapani et al. 2016). It is conceivable that ontogenic resistance is delayed or impaired when the progression of organ maturation is blocked by Rhodococcus fascians.

\section{CONCLUSIONS AND FUTURE DIRECTIONS}

Studies in the last decade have provided insights into the molecular mechanisms behind various types of age-dependent defense response. With the identification of key genes and regulatory cascades, we now have tools to decipher this complex phenomenon that integrates environmental cues, host physiology and pathogen life cycle. The newly discovered ARR components can serve as candidate molecular markers of plant age to guide the timing of pesticide application. These ARR genes could also be genetically engineered and used in molecular breeding for broadspectrum disease resistance. Introducing ARR genes into normally incompetent developmental stages can be a strategy to enhance disease resistance with low negative consequence. Some efforts are being made in this direction (Rinaldo et al. 2017).

The molecular links that integrate developmental timing and ARR activation is leading to some important themes in ARR-related research. First, morphological alterations and ARR onset associated with plant maturation is genetically decouplable. This can be achieved by assigning tissue specific development or defense function to a master regulator. Second, ARR involves a collection of developmentally acquired consolidation in disease resistance including physical barriers, chemical defense and innate immunity. Third, ARR components are targeted by pathogen-derived virulence factors to dampen host immunity. Therefore, effectors can be useful probes to dissect the mechanisms of ARR.

Exciting discoveries also lead to new challenges. Many ARR genes are conserved regulators of developmental transitions. Defense genes usually undergo fast evolution to escape and combat pathogen targeting. How do plants solve this paradox? Is the defense or development function the more ancient role of these genes? How plants measure time to activate ARR. Neither the environmental nor the intrinsic cues triggering ARR have been elucidated. Do plants use the same set of signals for developmental transition and ARR, or are there unknown biotic signals to set the timing of ARR? Last but not least, phytohormones are versatile regulators of plant development and defense. How does hormonal crosstalk occur in an age-dependent manner for disease resistance? Dissecting the fundamental mechanisms for crosstalk between development and immunity provides an exciting direction for future research.

\section{ACKNOWLEDGMENTS}

We thank members of the Yang lab for critical reading and discussion.

\section{LITERATURE CITED}

Abedon, B., and Tracy, W. 1996. Corngrass 1 of maize (Zea mays L.) delays development of adult plant resistance to common rust (Puccinia sorghi Schw.) and European corn borer (Ostrinia nubilalis Hubner). J. Hered. 87: 219-223.

Alabi, O. J., Zheng, Y., Jagadeeswaran, G., Sunkar, R., and Naidu, R. A. 2012. High-throughput sequence analysis of small RNA $\mathrm{s}$ in grapevine (Vitis vinifera L.) affected by grapevine leafroll disease. Mol. Plant Pathol. 13: 1060-1076.

Ando, K., Carr, K. M., Colle, M., Mansfeld, B. N., and Grumet, R. 2015. Exocarp properties and transcriptomic analysis of cucumber (Cucumis sativus) fruit expressing age-related resistance to Phytophthora capsici. PLoS One 10:e0142133.

Asalf, B., Gadoury, D. M., Tronsmo, A. M., Seem, R. C., Dobson, A., Peres, N. A., and Stensvand, A. 2014. Ontogenic resistance of leaves and fruit, and how leaf folding influences the distribution of powdery mildew on strawberry plants colonized by Podosphaera aphanis. Phytopathology 104: 954-963.

Baqui, M., and Kershaw, W. 1993. Effect of plant age on host preference, honeydew production and fecundity of Nilaparvata lugens (Stål) (Hom., Delphacidae) on rice cultivars. J. Appl. Entomol. 116:133-138.

Beydler, B. D., Osadchuk, K., Cheng, C.-L., Manak, J. R., and Irish, E. E. 2016. The juvenile phase of maize sees upregulation of stress-response genes and is extended by exogenous JA. Plant Physiol. 01707.02015.

Birkenbihl, R. P., Jach, G., Saedler, H., and Huijser, P. 2005. Functional dissection of the plant-specific SBP-domain: Overlap of the DNA-binding and nuclear localization domains. J. Mol. Biol. 352:585-596.

Bomblies, K., and Weigel, D. 2007. Hybrid necrosis: Autoimmunity as a potential gene-flow barrier in plant species. Nat. Rev. Genet. 8:382-393.

Brown, J. K. 2002. Yield penalties of disease resistance in crops. Curr. Opin. Plant Biol. 5:339-344.

Cardon, G., Höhmann, S., Klein, J., Nettesheim, K., Saedler, H., and Huijser, P. 1999. Molecular characterisation of the Arabidopsis SBP-box genes. Gene 237:91-104.

Century, K. S., Lagman, R. A., Adkisson, M., Morlan, J., Tobias, R., Schwartz, K., Smith, A., Love, J., Ronald, P. C., and Whalen, M. C. 1999. Developmental control of $\mathrm{Xa21}$-mediated disease resistance in rice. Plant $\mathrm{J}$. 20:231-236.

Chandler, M., and Tracy, W. 2007. Vegetative phase change among sweet corn (Zea mays L.) hybrids varying for reaction to common rust (Puccinia sorghi Schw.). Plant Breed. 126:569-573.

Chang, S. H., Tan, C. M., Wu, C. T., Lin, T. H., Jiang, S. Y., Liu, R. C., Tsai, M. C., Su, L. W., and Yang, J. Y. 2018. Alterations of plant architecture and phase transition by the phytoplasma virulence factor SAP11. J. Exp. Bot. 69:5389-5401.

Chuck, G., Cigan, A. M., Saeteurn, K., and Hake, S. 2007. The heterochronic maize mutant Corngrass1 results from overexpression of a tandem microRNA. Nat. Genet. 39:544-549.

Coelho, P. S., Valério, L., and Monteiro, A. A. 2009. Leaf position, leaf age and plant age affect the expression of downy mildew resistance in Brassica oleracea. Eur. J. Plant Pathol. 125:179-188.

Cui, H., Tsuda, K., and Parker, J. E. 2015. Effector-triggered immunity: from pathogen perception to robust defense. Annu. Rev. Plant Biol. 66: 487-511.

Cui, M., Wang, C., Zhang, W., Pervaiz, T., Haider, M. S., Tang, W., and Fang, J. 2018. Characterization of $V_{v}-m i R 156: V v-S P L$ pairs involved in the modulation of grape berry development and ripening. Mol. Genet. Genomics 293:1333-1354.

Depuydt, S., De Veylder, L., Holsters, M., and Vereecke, D. 2009. Eternal youth, the fate of developing Arabidopsis leaves upon Rhodococcus fascians infection. Plant Physiol. 149:1387-1398. 
Develey-Riviere, M. P., and Galiana, E. 2007. Resistance to pathogens and host developmental stage: A multifaceted relationship within the plant kingdom. New Phytol. 175:405-416.

Dhandapani, P., Song, J., Novak, O., and Jameson, P. E. 2016. Infection by Rhodococcus fascians maintains cotyledons as a sink tissue for the pathogen. Ann. Bot. (Lond.) 119:841-852.

Fabro, G., Steinbrenner, J., Coates, M., Ishaque, N., Baxter, L., Studholme, D. J., Körner, E., Allen, R. L., Piquerez, S. J., and Rougon-Cardoso, A. 2011. Multiple candidate effectors from the oomycete pathogen Hyaloperonospora arabidopsidis suppress host plant immunity. PLoS Pathog 7: e1002348.

Fouracre, J. P., and Poethig, R. S. 2016. The role of small RNAs in vegetative shoot development. Curr. Opin. Plant Biol. 29:64-72.

Gai, Y.-P., Li, Y.-Q., Guo, F.-Y., Yuan, C.-Z., Mo, Y.-Y., Zhang, H.-L., Wang, H., and Ji, X.-L. 2014. Analysis of phytoplasma-responsive sRNAs provide insight into the pathogenic mechanisms of mulberry yellow dwarf disease. Sci. Rep. 4:5378.

Ge, Y., Han, J., Zhou, G., Xu, Y., Ding, Y., Shi, M., Guo, C., and Wu, G. 2018. Silencing of miR156 confers enhanced resistance to brown planthopper in rice. Planta 248:813-826.

Glander, S., He, F., Schmitz, G., Witten, A., Telschow, A., and de Meaux, J. 2018. Assortment of flowering time and immunity alleles in natural Arabidopsis thaliana populations suggests immunity and vegetative lifespan strategies coevolve. Genome Biol. Evol. 10:2278-2291.

Gusberti, M., Gessler, C., and Broggini, G. A. 2013. RNA-Seq analysis reveals candidate genes for ontogenic resistance in Malus-Venturia pathosystem. PLoS One 8:e78457.

He, J., Xu, M., Willmann, M. R., McCormick, K., Hu, T., Yang, L., Starker, C. G., Voytas, D. F., Meyers, B. C., and Poethig, R. S. 2018. Thresholddependent repression of SPL gene expression by miR156/miR157 controls vegetative phase change in Arabidopsis thaliana. PLoS Genet. 14: e1007337

Herrera-Foessel, S. A., Singh, R. P., Lillemo, M., Huerta-Espino, J., Bhavani, S., Singh, S., Lan, C., Calvo-Salazar, V., and Lagudah, E. S. 2014. Lr67/ Yr46 confers adult plant resistance to stem rust and powdery mildew in wheat. Theor. Appl. Genet. 127:781-789.

Hershman, D., Hendrix, J., Stuckey, R., Bachi, P., and Henson, G. 1990. Influence of planting date and cultivar on soybean sudden death syndrome in Kentucky. Plant Dis. 74:761-766.

Hesler, L. S., Riedell, W. E., Langham, M. A., and Osborne, S. L. 2005. Insect infestations, incidence of viral plant diseases, and yield of winter wheat in relation to planting date in the northern Great Plains. J. Econ. Entomol. 98: 2020-2027.

Howe, G. A., and Jander, G. 2008. Plant immunity to insect herbivores. Annu. Rev. Plant Biol. 59:41-66.

Huang, X.-Q., and Röder, M. S. 2004. Molecular mapping of powdery mildew resistance genes in wheat: A review. Euphytica 137:203-223.

Huijser, P., and Schmid, M. 2011. The control of developmental phase transitions in plants. Development 138:4117-4129.

Hunger, R., Sherwood, J., Evans, C., and Montana, J. 1992. Effects of planting date and inoculation date on severity of wheat streak mosaic in hard red winter wheat cultivars. Plant Dis. 76:1056-1060.

Huot, B., Yao, J., Montgomery, B. L., and He, S. Y. 2014. Growth-defense tradeoffs in plants: a balancing act to optimize fitness. Mol. Plant 7: 1267-1287.

Hyun, Y., Richter, R., Vincent, C., Martinez-Gallegos, R., Porri, A., and Coupland, G. 2016. Multi-layered regulation of SPL15 and cooperation with SOC1 integrate endogenous flowering pathways at the Arabidopsis shoot meristem. Dev. Cell 37:254-266.

Jones, J. D., and Dangl, J. L. 2006. The plant immune system. Nature 444: 323-329.

Jones, J. D., Vance, R. E., and Dangl, J. L. 2016. Intracellular innate immune surveillance devices in plants and animals. Science 354:aaf6395.

Kimura, Y., Aoki, S., Ando, E., Kitatsuji, A., Watanabe, A., Ohnishi, M., Takahashi, K., Inoue, S.-i., Nakamichi, N., and Tamada, Y. 2015. A flowering integrator, SOC1, affects stomatal opening in Arabidopsis thaliana. Plant Cell Physiol. 56:640-649.

Korves, T. M. 2003. A developmental response to pathogen infection in Arabidopsis. Plant Physiol. 133:339-347.

Li, Y., Lu, Y.-G., Shi, Y., Wu, L., Xu, Y.-J., Huang, F., Guo, X.-Y., Zhang, Y., Fan, J., and Zhao, J.-Q. 2014. Multiple rice microRNAs are involved in immunity against the blast fungus Magnaporthe oryzae. Plant Physiol. 164: 1077-1092.

Li, Y., Zhang, Q., Zhang, J., Wu, L., Qi, Y., and Zhou, J.-M. 2010. Identification of microRNAs involved in pathogen-associated molecular patterntriggered plant innate immunity. Plant Physiol. 152:2222-2231.

Liang, X., Nazarenus, T. J., and Stone, J. M. 2008. Identification of a consensus DNA-binding site for the Arabidopsis thaliana SBP domain tran- scription factor, AtSPL14, and binding kinetics by surface plasmon resonance. Biochemistry 47:3645-3653.

Liu, C., Chen, H., Er, H. L., Soo, H. M., Kumar, P. P., Han, J.-H., Liou, Y. C., and $\mathrm{Yu}, \mathrm{H}$. 2008. Direct interaction of AGL24 and SOC1 integrates flowering signals in Arabidopsis. Development 135:1481-1491.

Liu, C., Thong, Z., and Yu, H. 2009. Coming into bloom: The specification of floral meristems. Development 136:3379-3391.

Liu, M., Shi, Z., Zhang, X., Wang, M., Zhang, L., Zheng, K., Liu, J., Hu, X., Di, C., and Qian, Q. 2019. Inducible overexpression of Ideal Plant Architecture 1 improves both yield and disease resistance in rice. Nat. Plants 5: $389-400$

Luan, Y., Cui, J., Li, J., Jiang, N., Liu, P., and Meng, J. 2018. Effective enhancement of resistance to Phytophthora infestans by overexpression of miR172a and b in Solanum lycopersicum. Planta 247:127-138.

Lyons, R., Rusu, A., Stiller, J., Powell, J., Manners, J. M., and Kazan, K. 2015. Investigating the association between flowering time and defense in the Arabidopsis thaliana-Fusarium oxysporum interaction. PLoS One 10: e0127699.

Mansfeld, B. N., Colle, M., Kang, Y., Jones, A. D., and Grumet, R. 2017. Transcriptomic and metabolomic analyses of cucumber fruit peels reveal a developmental increase in terpenoid glycosides associated with age-related resistance to Phytophthora capsici. Hortic. Res. 4:17022.

Mao, Y. B., Liu, Y. Q., Chen, D. Y., Chen, F. Y., Fang, X., Hong, G. J., Wang, L. J., Wang, J. W., and Chen, X. Y. 2017. Jasmonate response decay and defense metabolite accumulation contributes to age-regulated dynamics of plant insect resistance. Nat. Commun. 8:13925.

Marla, S. R., Chu, K., Chintamanani, S., Multani, D. S., Klempien, A., DeLeon, A., Bong-Suk, K., Dunkle, L. D., Dilkes, B. P., and Johal, G. S. 2018. Adult plant resistance in maize to northern leaf spot is a feature of partial loss-of-function alleles of Hm1. PLoS Pathog. 14:e1007356.

McDowell, J. M., Williams, S. G., Funderburg, N. T., Eulgem, T., and Dangl, J. L. 2005. Genetic analysis of developmentally regulated resistance to downy mildew (Hyaloperonospora parasitica) in Arabidopsis thaliana. Mol. Plant-Microbe Interact. 18:1226-1234.

Melotto, M., Underwood, W., Koczan, J., Nomura, K., and He, S. Y. 2006. Plant stomata function in innate immunity against bacterial invasion. Cell 126:969-980.

Moschini, R., and Pérez, B. 1999. Predicting wheat leaf rust severity using planting date, genetic resistance, and weather variables. Plant Dis. 83: 381-384.

Moyroud, E., Kusters, E., Monniaux, M., Koes, R., and Parcy, F. 2010. LEAFY blossoms. Trends Plant Sci. 15:346-352.

Mukhtar, M. S., Carvunis, A. R., Dreze, M., Epple, P., Steinbrenner, J., Moore, J., Tasan, M., Galli, M., Hao, T., Nishimura, M. T., Pevzner, S. J., Donovan, S. E., Ghamsari, L., Santhanam, B., Romero, V., Poulin, M. M., Gebreab, F., Gutierrez, B. J., Tam, S., Monachello, D., Boxem, M., Harbort, C. J., McDonald, N., Gai, L., Chen, H., He, Y., European Union Effectoromics, C., Vandenhaute, J., Roth, F. P., Hill, D. E., Ecker, J. R., Vidal, M., Beynon, J., Braun, P., and Dangl, J. L. 2011. Independently evolved virulence effectors converge onto hubs in a plant immune system network. Science 333: 596-601.

Padmanabhan, M. S., Ma, S., Burch-Smith, T. M., Czymmek, K., Huijser, P., and Dinesh-Kumar, S. P. 2013. Novel positive regulatory role for the SPL6 transcription factor in the N TIR-NB-LRR receptor-mediated plant innate immunity. PLoS Pathog. 9:e1003235.

Panter, S. N., Hammond-Kosack, K. E., Harrison, K., Jones, J. D., and Jones, D. A. 2002. Developmental control of promoter activity is not responsible for mature onset of Cf-9B-mediated resistance to leaf mold in tomato. Mol. Plant-Microbe Interact. 15:1099-1107.

Panter, S., and Jones, D. A. 2002. Age-related resistance to plant pathogens. Adv. Bot. Res. 38:251-280.

Patharkar, O. R., Gassmann, W., and Walker, J. C. 2017. Leaf shedding as an anti-bacterial defense in Arabidopsis cauline leaves. PLoS Genet. 13: e1007132.

Pertry, I., Václavíková, K., Depuydt, S., Galuszka, P., Spíchal, L., Temmerman, W., Stes, E., Schmülling, T., Kakimoto, T., and Van Montagu, M. C. 2009. Identification of Rhodococcus fascians cytokinins and their modus operandi to reshape the plant. Proc. Natl. Acad. Sci. 106:929-934

Poethig, R. S. 2013. Vegetative phase change and shoot maturation in plants.Curr. Top. Dev. Biol. 105:125-152.

Rhoades, M. W., Reinhart, B. J., Lim, L. P., Burge, C. B., Bartel, B., and Bartel, D. P. 2002. Prediction of plant microRNA targets. Cell 110: 513-520.

Rinaldo, A., Gilbert, B., Boni, R., Krattinger, S. G., Singh, D., Park, R. F., Lagudah, E., and Ayliffe, M. 2017. The Lr34 adult plant rust resistance gene provides seedling resistance in durum wheat without senescence. Plant Biotechnol. J. 15:894-905. 
Risk, J. M., Selter, L. L., Krattinger, S. G., Viccars, L. A., Richardson, T. M., Buesing, G., Herren, G., Lagudah, E. S., and Keller, B. 2012. Functional variability of the $L r 34$ durable resistance gene in transgenic wheat. Plant Biotechnol. J. 10:477-487.

Robert-Seilaniantz, A., Grant, M., and Jones, J. D. 2011. Hormone crosstalk in plant disease and defense: More than just jasmonate-salicylate antagonism. Annu. Rev. Phytopathol. 49:317-343.

Ross, A. F. 1961. Systemic acquired resistance induced by localized virus infections in plants. Virology 14:340-358.

Rusterucci, C., Zhao, Z., Haines, K., Mellersh, D., Neumann, M., and Cameron, R. K. 2005. Age-related resistance to Pseudomonas syringae pv. tomato is associated with the transition to flowering in Arabidopsis and is effective against Peronospora parasitica. Physiol. Mol. Plant Pathol. 66:222-231.

Saijo, Y., Loo, E. P. i., and Yasuda, S. 2018. Pattern recognition receptors and signaling in plant-microbe interactions. Plant J. 93:592-613.

Saur, I. M., Kadota, Y., Sklenar, J., Holton, N. J., Smakowska, E., Belkhadir, Y., Zipfel, C., and Rathjen, J. P. 2016. NbCSPR underlies age-dependent immune responses to bacterial cold shock protein in Nicotiana benthamiana. Proc. Natl. Acad. Sci. USA 113:3389-3394.

Singh, R. P., Huerta-Espino, J., and William, H. M. 2005. Genetics and breeding for durable resistance to leaf and stripe rusts in wheat. Turk. J. Agric. For. 29:121-127.

Steffenson, B., Hayes, P., and Kleinhofs, A. 1996. Genetics of seedling and adult plant resistance to net blotch (Pyrenophora teres $\mathrm{f}$. teres) and spot blotch (Cochliobolus sativus) in barley. Theor. Appl. Genet. 92:552-558.

Torres, D. P., Proels, R. K., Schempp, H., and Huckelhoven, R. 2017. Silencing of RBOHF2 causes leaf age-dependent accelerated senescence, salicylic acid accumulation, and powdery mildew resistance in barley. Mol. PlantMicrobe Interact. 30:906-918.

Toruño, T. Y., Stergiopoulos, I., and Coaker, G. 2016. Plant-pathogen effectors: Cellular probes interfering with plant defenses in spatial and temporal manners. Annu. Rev. Phytopathol. 54:419-441.

Uauy, C., Brevis, J. C., Chen, X., Khan, I., Jackson, L., Chicaiza, O., Distelfeld, A., Fahima, T., and Dubcovsky, J. 2005. High-temperature adultplant (HTAP) stripe rust resistance gene Yr36 from Triticum turgidum ssp. dicoccoides is closely linked to the grain protein content locus Gpc-B1. Theor. Appl. Genet. 112:97.

Vereecke, D., Burssens, S., Simón-Mateo, C., Inzé, D., Van Montagu, M., Goethals, K., and Jaziri, M. 2000. The Rhodococcus fascians-plant interaction: Morphological traits and biotechnological applications. Planta 210:241-251.

Wagner, D. 2017. Key developmental transitions during flower morphogenesis and their regulation. Curr. Opin. Genet. Dev. 45:44-50.

Wang, H., and Wang, H. 2015. The miR156/SPL module, a regulatory hub and versatile toolbox, gears up crops for enhanced agronomic traits. Mol. Plant 8:677-688.

Wang, J.-W., Czech, B., and Weigel, D. 2009. miR156-regulated SPL transcription factors define an endogenous flowering pathway in Arabidopsis thaliana. Cell 138:738-749.

Weßling, R., Epple, P., Altmann, S., He, Y., Yang, L., Henz, S. R., McDonald, N., Wiley, K., Bader, K. C., and Gläßer, C. 2014. Convergent targeting of a common host protein-network by pathogen effectors from three kingdoms of life. Cell Host Microbe 16:364-375.

Whalen, M. C. 2005. Host defence in a developmental context. Mol. Plant Pathol. 6:347-360

Wilson, D. C., Carella, P., Isaacs, M., and Cameron, R. K. 2013. The floral transition is not the developmental switch that confers competence for the Arabidopsis age-related resistance response to Pseudomonas syringae pv. tomato. Plant Mol. Biol. 83:235-246.

Wilson, D. C., Kempthorne, C. J., Carella, P., Liscombe, D. K., and Cameron, R. K. 2017. Age-related resistance in Arabidopsis thaliana involves the
MADS-domain transcription factor SHORT VEGETATIVE PHASE and direct action of salicylic acid on Pseudomonas syringae. Mol. PlantMicrobe Interact. 30:919-929.

Winter, C. M., Austin, R. S., Blanvillain-Baufume, S., Reback, M. A., Monniaux, M., Wu, M. F., Sang, Y., Yamaguchi, A., Yamaguchi, N., Parker, J. E., Parcy, F., Jensen, S. T., Li, H., and Wagner, D. 2011. LEAFY target genes reveal floral regulatory logic, cis motifs, and a link to biotic stimulus response. Dev. Cell 20:430-443.

Wu, G., Park, M. Y., Conway, S. R., Wang, J.-W., Weigel, D., and Poethig, R. S. 2009. The sequential action of miR156 and miR172 regulates developmental timing in Arabidopsis. Cell 138:750-759.

Wyatt, S., and Kuc, J. 1992. The effect of leaf age, flowering, and senescence on the resistance of tobacco to blue mold. Phytopathology 80:1000.

Xie, K., Shen, J., Hou, X., Yao, J., Li, X., Xiao, J., and Xiong, L. 2012. Gradual increase of miR156 regulates temporal expression changes of numerous genes during leaf development in rice. Plant Physiol. 158: $1382-1394$.

Xu, M., Hu, T., Zhao, J., Park, M.-Y., Earley, K. W., Wu, G., Yang, L., and Poethig, R. S. 2016. Developmental functions of miR156-regulated SQUAMOSA PROMOTER BINDING PROTEIN-LIKE (SPL) genes in Arabidopsis thaliana. PLoS Genet. 12:e1006263.

Xu, Y. P., Lv, L. H., Xu, Y. J., Yang, J., Cao, J. Y., and Cai, X. Z. 2018. Leaf stage-associated resistance is correlated with phytohormones in a pathosystem-dependent manner. J. Integr. Plant Biol. 60:703-722.

Yamaguchi, A., Wu, M.-F., Yang, L., Wu, G., Poethig, R. S., and Wagner, D. 2009. The microRNA-regulated SBP-Box transcription factor SPL3 is a direct upstream activator of LEAFY, FRUITFULL, and APETALA1. Dev. Cell 17:268-278.

Yang, C.-Y., Huang, Y.-H., Lin, C.-P., Lin, Y.-Y., Hsu, H.-C., Wang, C.-N., Li-Yu, D. L., Shen, B.-N., and Lin, S.-S. 2015. MicroRNA396-targeted SHORT VEGETATIVE PHASE is required to repress flowering and is related to the development of abnormal flower symptoms by the phyllody symptoms1 effector. Plant Physiol. 168:1702-1716.

Yin, H., Hong, G., Li, L., Zhang, X., Kong, Y., Sun, Z., Li, J., Chen, J., and He, Y. 2019. miR156/SPL9 regulates reactive oxygen species accumulation and immune response in Arabidopsis thaliana. Phytopathology 109:632-642.

Zhang, C., Liu, L., Wang, X., Vossen, J., Li, G., Li, T., Zheng, Z., Gao, J., Guo, Y., and Visser, R. G. 2014. The Ph-3 gene from Solanum pimpinellifolium encodes CC-NBS-LRR protein conferring resistance to Phytophthora infestans. Theor. Appl. Genet. 127:1353-1364.

Zhang, W., Gao, S., Zhou, X., Chellappan, P., Chen, Z., Zhou, X., Zhang, X., Fromuth, N., Coutino, G., and Coffey, M. 2011. Bacteria-responsive microRNAs regulate plant innate immunity by modulating plant hormone networks. Plant Mol. Biol. 75:93-105.

Zheng, X.-y., Zhou, M., Yoo, H., Pruneda-Paz, J. L., Spivey, N. W., Kay, S. A., and Dong, X. 2015. Spatial and temporal regulation of biosynthesis of the plant immune signal salicylic acid. Proc. Natl. Acad. Sci. 112: 9166-9173.

Zhu, Q.-H., and Helliwell, C. A. 2010. Regulation of flowering time and floral patterning by miR172. J. Exp. Bot. 62:487-495.

Zipfel, C. 2014. Plant pattern-recognition receptors. Trends Immunol. 35: 345-351.

Zipfel, C., Robatzek, S., Navarro, L., Oakeley, E. J., Jones, J. D., Felix, G., and Boller, T. 2004. Bacterial disease resistance in Arabidopsis through flagellin perception. Nature 428:764-767.

Zou, Y., Wang, S., Zhou, Y., Bai, J., Huang, G., Liu, X., Zhang, Y., Tang, D., and Lu, D. 2018. Transcriptional regulation of the immune receptor FLS2 controls the ontogeny of plant innate immunity. The Plant Cell 30: 2779-2794. 